\title{
A New PMU-Based Fault Detection/Location Technique for Transmission Lines With Consideration of Arcing Fault Discrimination-Part I: Theory and Algorithms
}

\author{
Ying-Hong Lin, Member, IEEE, Chih-Wen Liu, Senior Member, IEEE, and Ching-Shan Chen, Student Member, IEEE
}

\begin{abstract}
A new fault detection/location technique with consideration of arcing fault discrimination based on phasor measurement units for extremely high voltage/ultra-high voltage transmission lines is presented in this two-paper set. Part I of this two-paper set is mainly aimed at theory and algorithm derivation. The proposed fault detection technique for both arcing and permanent faults is achieved by a combination of a fault detection index $|M|$ and a fault location index $|D|$, which are obtained by processing synchronized fundamental phasors. One is to detect the occurrence of a fault and the other is to distinguish between in-zone and out-ofzone faults. Furthermore, for discriminating between arcing and permanent faults, the proposed technique estimates the amplitude of arc voltage by least error squares method through the measured synchronized harmonic phasors caused by the nonlinear arc behavior. Then, the discrimination will be achieved by comparing the estimated amplitude of arc voltage to a given threshold value. In addition, in order to eliminate the error caused by exponentially decaying dc offset on the computations of fundamental and harmonic phasors, an extended discrete Fourier transform algorithm is also presented.
\end{abstract}

Index Terms-Arcing fault discrimination, extended discrete Fourier transform (EDFT), fault detection, fault location, phasor measurement unit (PMU).

\section{INTRODUCTION}

I N POWER SYSTEMS, high-voltage transmission lines are vital links that achieve the essential continuity of service from generating plants to end users. Over the past 20 years, many studies have been done on the transmission-line protection including fault detection/location and arcing fault discrimination for avoiding reclosing on a permanent fault [1]-[12]. The majority of investigations on fault detection/location are concerned with permanent faults [1]-[6], [11], [12]. However, most of the faults on extremely high voltage (EHV)/ultra-high voltage (UHV) overhead transmission lines are temporary fault (arcing

Manuscript received January 6, 2003; revised May 20, 2003. Paper no. TPWRD-00001-2003. This work was supported by the National Science Council of the Republic of China under Contract NSC91-2213-E-309-006.

Y.-H. Lin is with the Department of Engineering and Management of Advanced Technology, Chang Jung Christian University, Tainan 711, Taiwan, R.O.C. (e-mail: inhon@mail.cju.edu.tw).

C.-W. Liu and C.-S. Chen are with the Department of Electrical Engineering, National Taiwan University, Taipei 106, Taiwan, R.O.C. (e-mail: cwliu@cc.ee.ntu.edu.tw; d89921019@ms89.ntu.edu.tw).

Digital Object Identifier 10.1109/TPWRD.2004.832407 fault) such as insulator flashover... etc. Recently, Djuric et al. took the arcing fault into account for estimation of fault location [7].

The arcing fault on the transmission lines can be cleared by momentarily de-energizing the faulted lines. Automatic reclosing is one of the economical and effective means to clear a temporary fault for improving the quality of customer power supply and system stability [1]. Nevertheless, reclosing on a permanent fault is undesirable and it may aggravate the potential damage to the system and equipment. To avoid reclosing on the permanent faults, some techniques were developed in the past [7]-[10]. In [7] and [8], the arc voltage is modeled as a square waveshape in phase with the fault current and M.B. Djuric et al. proposed numerical algorithms for arcing fault detection on overhead lines in spectral and time domain. In [7], the proposed time-domain solution for single-phase arcing fault detection is favorable for short and single-end sourcing transmission lines due to neglecting the existence of line capacitance and suffering from infeed current. In [8], the presented spectral approach is suitable for symmetrical arcing fault detection only. In [9], three criteria for distinguishing between temporary and permanent faults are proposed by virtual of analyzing voltages on the opened phase conductor during the reclosing dead time. In [10], an adaptive single-pole autoreclosure (SPAR) technique was developed using three-layer neural networks trained by frequency-domain decomposition of features.

In this two-paper set, we present a phasor-measurement-unit (PMU)-based fault detection/location technique for both arcing and permanent faults. It is achieved by processing the fundamental frequency voltage and current phasors, and a spectral algorithm utilizing the synchronized harmonic phasors measured at both ends of lines is also proposed for arcing fault discrimination. The distributed parameter model of long transmission lines is adopted to develop these algorithms. For an arcing fault, the arc voltage is modeled as a square waveshape in phase with the arc current [7], [8], and the unknown amplitude of arc voltage is estimated by least error squares (LES) method. By comparing the estimated amplitude of arc voltage with a given threshold value, the reclosing is decided to perform or not. In addition, in order to eliminate the error caused by exponentially decaying dc offset on the computations of fundamental and harmonic phasors, an extended discrete Fourier transform (EDFT) algorithm 
is also presented. Utilizing EDFT enhances the convergence speed and accuracy on the fault location/detection and the estimation of amplitude of arc voltage.

\section{Dynamic Characteristics of the Fault ArC}

A long arc, typical of the power system fault, possesses the highly nonlinear characteristics influenced by a number of factors (e.g., the arc path, arc column geometry, etc.). Some studies have shown that the dynamic arc characteristics can be represented by the following arc equation [8], [13]-[15]:

$$
\frac{d g}{d t}=\frac{1}{\tau}(G-g)
$$

where $g$ is the time-varying arc conductance, $G$ is the stationary arc conductance, and $\tau$ is the time constant.

The unknown model parameters in (1) can be estimated from the field test data. Goda et al. [15] have experimentally obtained the voltage-ampere cyclogram, which defines the arc hysteresis effect in the test of high current fault arc in free air. There are two important features in the measured arc voltage and current: 1) The arc voltage is in phase with arc current, and 2) the nonlinear variation of the arc manifest itself into producing high-frequency components which, in turn, distorted the arc voltage waveform into a near squarewave. In this paper, the arc model presented in [7] and [8] is adopted for derivation of proposed algorithm and is described as follows.

\section{A. Time-Domain Characteristics [7], [8]}

The arc voltage would be approximately expressed as a squarewave and depicted in (2)

$$
v_{\operatorname{arc}}(t)=V_{a} \operatorname{sign}\left(i_{\operatorname{arc}}(t)\right)
$$

where $v_{\text {arc }}(t)$ and $i_{\text {arc }}(t)$ are arc voltage and current, respectively, and $V_{a}$ is the amplitude of arc voltage. The function sign is defined as $\operatorname{sign}(x)=1$ if $x \geq 0, \operatorname{sign}(x)=-1$, if $x<0$. The value of $V_{a}$ is obtained from the product of arc-voltage gradient and the length of the path. Fig. 1 shows the computer simulated arc voltage and current waveforms.

\section{B. Spectrum-Domain Characteristics [8]}

The square wave has an important feature in the spectral domain. Namely, Fourier series containing odd sine components only as following can represent the square wave

$$
v_{\text {arc }}(t)=\sum_{n=1,3,5 \ldots}^{\infty} \frac{4}{\pi} \frac{1}{n} V_{a} \sin \left(n \omega t+\theta_{n}\right)
$$

where $n, \omega$, and $\theta_{n}$ are the order of harmonic, fundamental angular frequency and phase angle of the $n$th harmonic, respectively.

Therefore, the phasor of the $n$th harmonic is expressed as

$$
V_{\mathrm{arc}, n}=\frac{4}{\pi} \frac{1}{n} V_{a} e^{j \theta_{n}}
$$

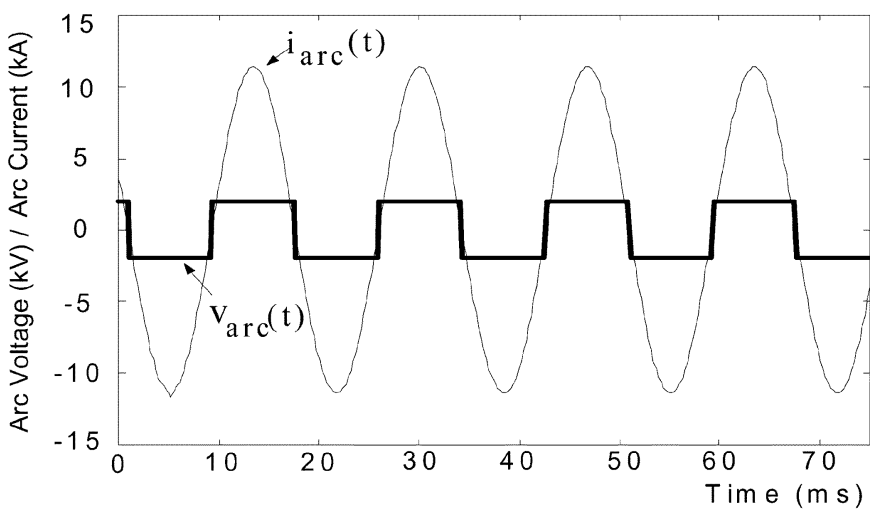

Fig. 1. Computer simulated arc voltage and current waveforms.

\section{Overall Configuration of The Proposed TeChNiQue}

To demonstrate the proposed technique, assume that an a-phase arcing ground fault occurs on the transmission lines at $\mathrm{D}$ (per unit) away from the receiving end as shown in Fig. 2.

The outsides of both ends of lines are replaced by Thevenin's equivalences and plotted in a one-line diagram for simplicity. The PMUs are equipped at both ends of the lines to synchronously measure three-phase fundamental and harmonic voltage and current phasors. In our previous paper [12], the design, implementation, and tests of the PMU have been detailed and the timing difference between two PMUs of less than $1 \mu$ s has been proven. The proposed algorithms combined with PMUs and communication links form the fault location/detection system with the consideration of arcing fault discrimination. In Fig. 2, the a-phase arcing fault is modeled as a serial connection of arc voltage and fault resistance. The flowchart of the proposed algorithm is shown in Fig. 3 and described as follows.

The synchronized phasors measured by PMUs will be transmitted to the central computer via communication channels. In order to reduce the burden of communication channels, the harmonic phasors will be transmitted to the central computer after a fault has been detected. For fault detection/location on the transmission lines, the three-phase fundamental voltage and current phasors are utilized and transformed by symmetrical transformation to decouple the coupling effect among interphases. Then, the fundamental positive-sequence phasors will be utilized to calculate the fault detection index $|M|$ and fault location index $|D|$. We utilize $|M|$ and $|D|$ to detect/locate a fault irrespective of arcing or permanent faults. The $|M|$ is utilized to detect the occurrence of a fault by comparing it with a small threshold value $|M|_{\text {th }}$, and the $|D|$ is utilized to discriminate between in-zone and out-of-zone faults by examining the value and convergence of four consecutive $|D| s$ after the occurrence of a fault. The proposed criteria to examine the value and convergence of four consecutive $|D| s$ are as follows: 1) All of them lie in the interval $(0,1)$, and 2$)$ the deviations of them are less than a given threshold value $|D|_{\mathrm{th}}$. Furthermore, it is notable that a transmission-line parameter estimation technique proposed in our previous papers [11], [12] is also utilized to reduce the error caused by the uncertainty of line parameters. When a fault is detected and located, the harmonic voltage phasors at fault point and the harmonic current phasors passing through the fault path can be computed through the measured harmonic phasors at 


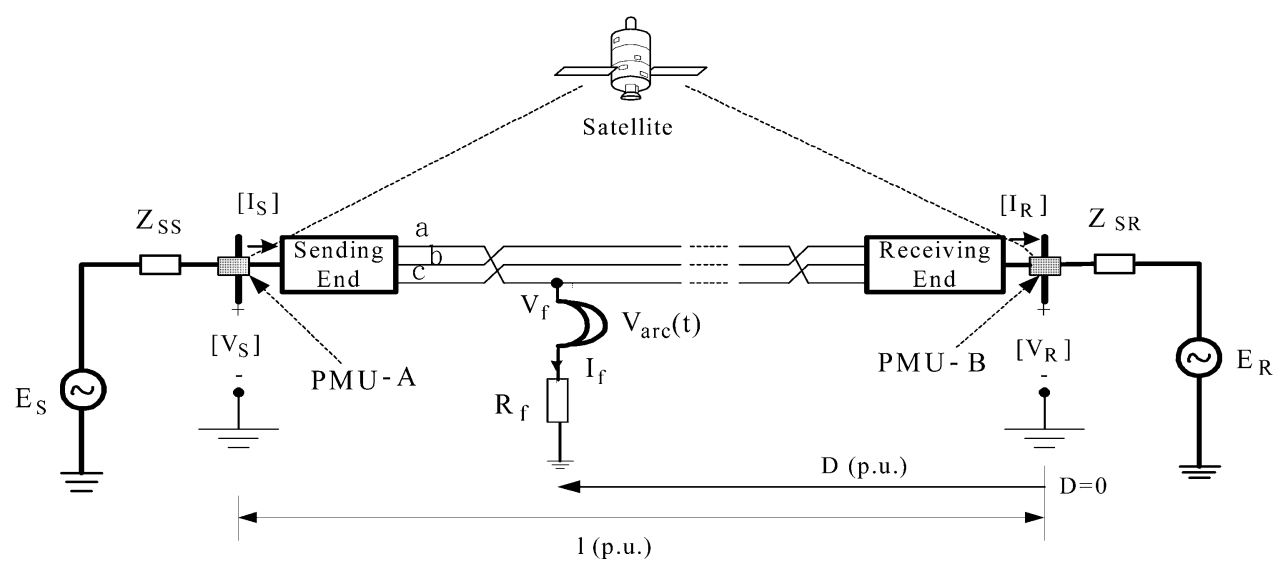

Fig. 2. Configuration of the fault detection/location system with the consideration of arcing fault discrimination.

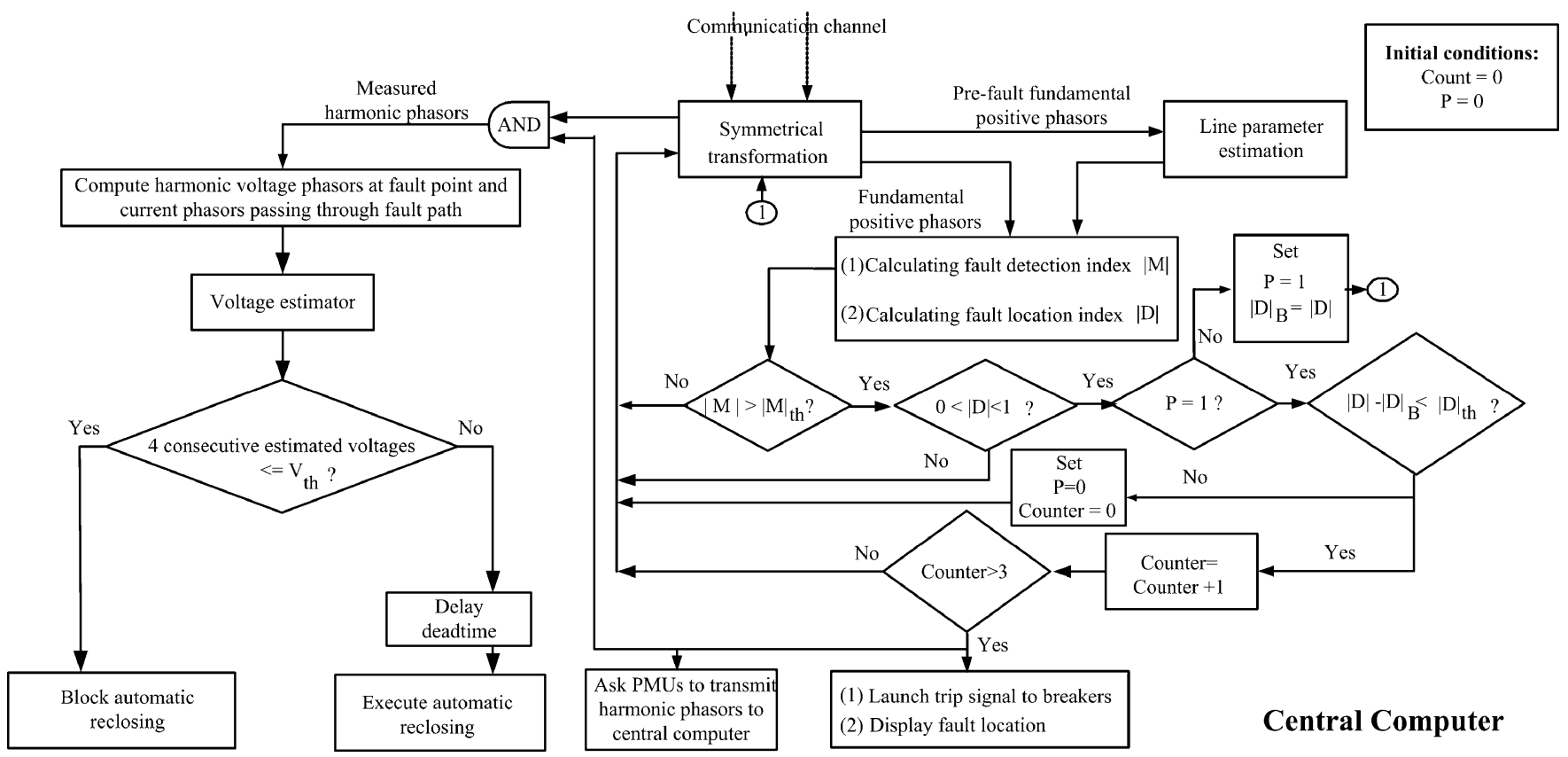

Fig. 3. Flowchart of fault detection/location technique with the consideration of arcing fault discrimination.

both ends of lines. Then, the proposed voltage estimator will estimate the amplitude of arc voltage. If four consecutive estimated voltages are not greater than a threshold value $V_{\mathrm{th}}$, the fault will be identified as a permanent (arcless) fault and the automatic reclosing should be blocked. Otherwise, the fault will be identified as an arcing fault and the automatic reclosing will be executed after deadtime.

\section{ALGORITHM DERIVATION}

The fault detection/location algorithm for a permanent fault has been presented in our previous papers [11], [12], and we utilize the same algorithm to detect/locate an arcing fault. Here, we only review briefly the fault detection/location algorithm and focus on arcing fault discrimination and extended DFT algorithm. The readers are encouraged to refer to [11] and [12] for detailed derivation of fault detection/location algorithm.

\section{A. Fault Detection/Location Algorithm}

Consider that a fault occurs on transposed three-phase transmission lines and the positive-sequence network of the faulted lines is shown in Fig. 4. The subscript 1 in Fig. 4 denotes the positive-sequence component.

The voltage $V_{f, 1}$ at fault point can be expressed in terms of two positive-sequence phasor sets $\left(V_{S, 1}, I_{S, 1}\right)$ and $\left(V_{R, 1}, I_{R, 1}\right)$. So the fault location index $|D|$ away from receiving end will be resolved easily and given in (5)

$$
D=\frac{\ln \left(\frac{N}{M}\right)}{2 \gamma_{1} \ell}
$$

where

$$
\begin{aligned}
& N=\frac{V_{R, 1}-\rho_{1} I_{R, 1}}{2}-\frac{V_{S, 1}-\rho_{1} I_{S, 1}}{2} \exp \left(\gamma_{1} \ell\right) \\
& M=\frac{V_{S, 1}+\rho_{1} I_{S, 1}}{2} \exp \left(-\gamma_{1} \ell\right)-\frac{V_{R, 1}+\rho_{1} I_{R, 1}}{2}
\end{aligned}
$$




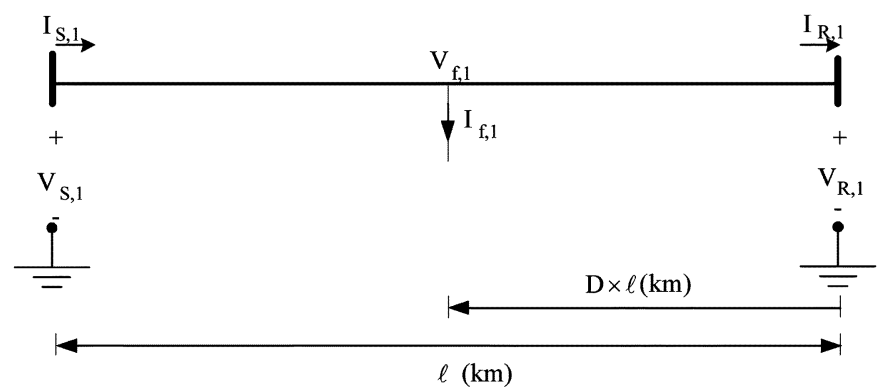

Fig. 4. Positive-sequence network of faulted lines.

where

$V_{R, 1}, V_{S, 1}$ positive-sequence voltage phasors at both ends of the lines;

$I_{R, 1}, I_{S, 1} \quad$ positive-sequence current phasors at both ends of the lines;

$\gamma_{1}, \rho_{1} \quad$ propagation constant and characteristic impedance in positive-sequence network, respectively;

$\ell \quad$ length of protected lines.

In this study, (5) will be utilized to locate both arcing and permanent faults. Moreover, it is remarkable that for untransposed lines, one can also find the transformation matrix for decoupling the coupling effect among interphases by eigenvalue/eigenvector theory [16]. Thus, (5) will be also used for untransposed transmission lines. In (7), the value $|M|$ is theoretically equal to zero under prefault condition and will increase rapidly as soon as a fault occurs. It possesses the great property to work as the fault detection index. The fault location index $|D|$ in (5) will converge fast to a value between 0 and 1 when an in-zone fault occurs. Nevertheless, it will be an undetermined value when an out-of-zone fault occurs. So it will be utilized to promote the security of fault detection.

\section{B. Derivation of Arcing Fault Discrimination Algorithm}

The key point of arcing fault discrimination is to estimate the amplitude of arc voltage described in (2). Then, discriminating between arcing and permanent faults will be achieved by comparing the estimated amplitude of arc voltage with a given threshold value. The algorithm for estimating amplitude of arc voltage is derived in following. It is based on an assumed condition that the fault type is known. Some practical fault-type classification schemes can be found in [2].

\section{Single-Phase Arcing Ground Fault}

Consider that an a-phase arcing ground fault occurs on transmission lines and the representation of the faulted lines is shown in Fig. 2. The arcing fault is modeled as a serial connection of arc voltage and fault resistance $R_{f}$. The faulted three-phase transmission lines shown in Fig. 2 can be represented by three decoupled single-phase faulted lines (i.e., positive-, negative-, and zero-sequence faulted lines, and they are shown in Figs. 5-7, respectively. In Figs. 5-7, the subscript $n$ denotes the $n$th harmonic phasors caused by an arcing fault, and 0,1 , and 2 denote the positive-, negative-, and zero-sequence components, respectively.

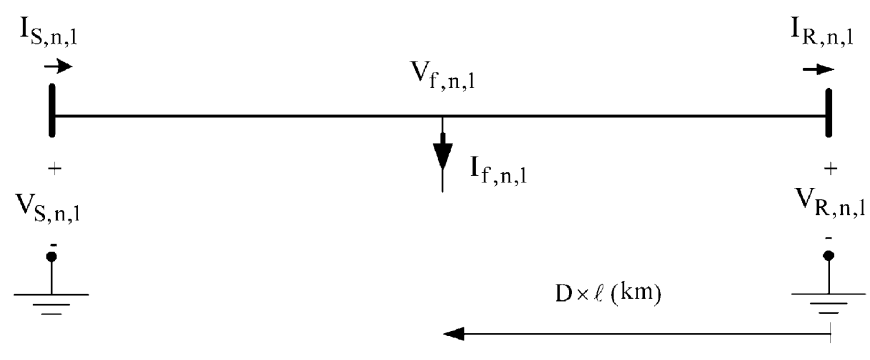

Fig. 5. Positive-sequence faulted line.

Since the fault location $D \times \ell$ is obtained by (5), one can calculate the harmonic voltage $V_{f, n}$ at fault point and the harmonic current $I_{f, n}$ passing through the fault path by taking the summation of positive-, negative-, and zero-sequence components. The $V_{f, n}$ and $I_{f, n}$ are given in (8) and (9)

$$
\begin{aligned}
& V_{f, n}=\sum_{k=0}^{2} V_{f, n, k} \\
& =\sum_{k=0}^{2}\left\{\frac{1}{2} \exp \left(-\gamma_{n, k}(\ell-D \ell)\right)\right. \\
& \times\left(V_{S, n, k}+\rho_{n, k} I_{S, n, k}\right) \\
& +\frac{1}{2} \exp \left(\gamma_{n, k}(\ell-D \ell)\right) \\
& \left.\times\left(V_{S, n, k}-\rho_{n, k} I_{S, n, k}\right)\right\} \\
& I_{f, n}=\sum_{k=0}^{2} I_{f, n, k} \\
& =\sum_{k=0}^{2}\left\{\frac{1}{2} \rho_{n, k}^{-1} e^{-\gamma_{n, k}(\ell-D \ell)}\left(V_{S, n, k}+\rho_{n, k} I_{S, n, k}\right)\right. \\
& -\frac{1}{2} \rho_{n, k}^{-1} e^{\gamma_{n, k}(\ell-D \ell)}\left(V_{S, n, k}-\rho_{n, k} I_{S, n, k}\right) \\
& +\frac{1}{2} \rho_{n, k}^{-1} e^{-\gamma_{n, k} D \ell}\left(V_{R, n, k}+\rho_{n, k} I_{R, n, k}\right) \\
& \left.-\frac{1}{2} \rho_{n, k}^{-1} e^{\gamma_{n, k} D \ell}\left(V_{R, n, k}-\rho_{n, k} I_{R, n, k}\right)\right\}
\end{aligned}
$$

where the subscript $n$ denotes the $n$th harmonic component, $k=0,1,2$ denote the zero-, positive-, and negative-sequence components, respectively, and $\gamma_{n}$ and $\rho_{n}$ are the propagation constant and characteristic impedance of transmission lines with the angular frequency of the $n$th harmonic, respectively.

So far, we have calculated the $V_{f, n}$ and $I_{f, n}$. Taking observation on Fig. 2 and (4), the relationship between $V_{f, n}$ and $I_{f, n}$ is given in (10)

$$
V_{f, n}=V_{\mathrm{arc}, n}+I_{f, n} R_{f} .
$$

After some algebraic manipulation in (10), we obtain

$$
\left|V_{f, n}-I_{f, n} R_{f}\right|=\left|V_{\mathrm{arc}, n}\right|
$$

where $|\bullet|$ denotes the magnitude of a complex number. 


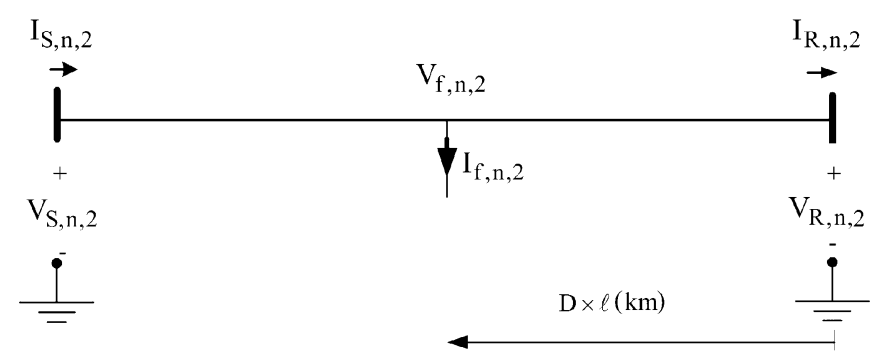

Fig. 6. Negative-sequence faulted line.

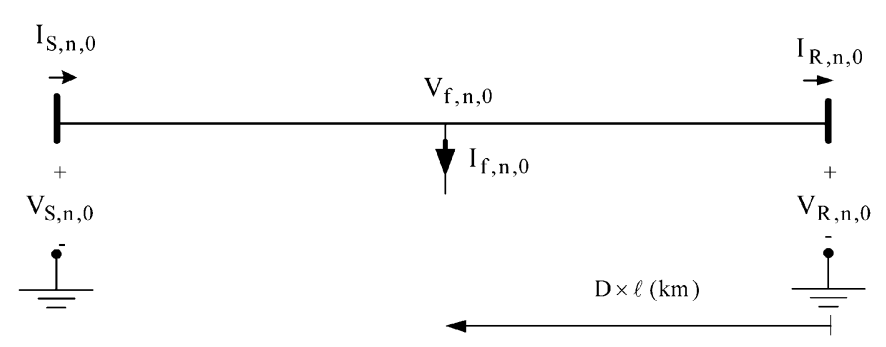

Fig. 7. Zero-sequence faulted line.

Combining (11) and (4), one can obtain

$$
\left|V_{f, n}\right|^{2}=\left(\frac{4}{n \pi}\right)^{2} V_{a}^{2}+2\left(V_{f, n} \circ I_{f, n}\right) R_{f}-\left|I_{f, n}\right|^{2} R_{f}^{2}
$$

where $\circ$ denotes inner product of two complex numbers.

If $p$ harmonic phasors are measured, (12) determines $p$ linear equations in three unknowns. For example, the third, fifth, seventh, and ninth harmonic phasors are measured, and then (12) will be written in matrix form as the following:

$$
\begin{aligned}
& {\left[\begin{array}{l}
\left|V_{f, 3}\right|^{2} \\
\left|V_{f, 5}\right|^{2} \\
\left|V_{f, 7}\right|^{2} \\
\left|V_{f, 9}\right|^{2}
\end{array}\right]=\left[\begin{array}{ccc}
\left(\frac{4}{3 \pi}\right)^{2} & 2\left(V_{f, 3} \circ I_{f, 3}\right) & -\left|I_{f, 3}\right|^{2} \\
\left(\frac{4}{5 \pi}\right)^{2} & 2\left(V_{f, 5} \circ I_{f, 5}\right) & -\left|I_{f, 5}\right|^{2} \\
\left(\frac{4}{7 \pi}\right)^{2} & 2\left(V_{f, 7} \circ I_{f, 7}\right) & -\left|I_{f, 7}\right|^{2} \\
\left(\frac{4}{9 \pi}\right)^{2} & 2\left(V_{f, 9} \circ I_{f, 9}\right) & -\left.I_{f, 9}\right|^{2}
\end{array}\right] } \\
& \times\left[\begin{array}{c}
V_{a}^{2} \\
R_{f} \\
R_{f}^{2}
\end{array}\right]+[\hat{\varepsilon}]
\end{aligned}
$$

where $[\hat{\varepsilon}]$ is a $4 \times 1$ error vector that represents the error caused by approximation of arc model and the measurement errors in $\mathrm{CT}$ and PT.

To write (13) in abbreviation from $\bar{B}=\bar{A} \bar{X}+\bar{\varepsilon}$, the unknown $\bar{X}$ can be estimated by means of the LES method in (14)

$$
\bar{X}=\left(\bar{A}^{t} \bar{A}\right)^{-1} \bar{A}^{t} \bar{B} .
$$

From the authors' experience, it is enough to take the third, fifth, seventh, and ninth harmonics into account for the estimation of $\bar{X}$, and the ninth harmonic is as redundancy. The fundamental frequency phasors are not applied to the estimation of $\bar{X}$ because the magnitudes of the fundamental frequency phasors are largely greater than the magnitudes of harmonics caused by an arcing fault. Applying fundamental frequency phasors to the LES method is easy to cause the ill condition in $\left(\bar{A}^{t} \bar{A}\right)^{-1}$.

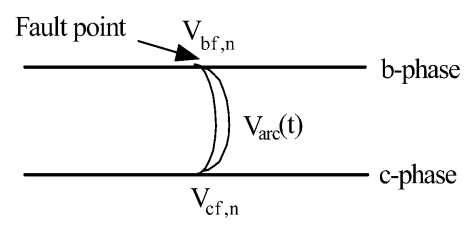

Fig. 8. BC-phase arcing fault.

\section{Symmetrical Arcing Fault}

For a symmetrical arcing fault (i.e., three-phase arcing ground fault), the linear equations as following are obtained by simplifying (12) with $R_{f}=0$ :

$$
\left|V_{f, n}\right|=\left(\frac{4}{n \pi}\right) V_{a} .
$$

Taking the third, fifth, seventh, and ninth harmonic phasors into account, (15) determines four linear equations in one unknown. The unknown $V_{a}$ can be estimated by means of the LES method too.

\section{E. Phase-to-Phase Arcing Fault}

For a bc-phase arcing fault shown in Fig. 8, one can calculate the differences of harmonic voltages between $b$-phase and c-phase at fault point. They are shown in the following equation:

$$
\begin{aligned}
V_{d f, n}= & V_{b f, n}-V_{c f, n} \\
= & \left(\alpha^{2}-\alpha\right) V_{f, n, 1}+\left(\alpha-\alpha^{2}\right) V_{f, n, 2} \\
= & \left(\alpha^{2}-\alpha\right)\left\{\frac{1}{2} e^{-\gamma_{n, 1}(\ell-D \ell)}\left(V_{S, n, 1}+\rho_{n, 1} I_{S, n, 1}\right)\right. \\
& \left.\quad+\frac{1}{2} e^{\gamma_{n, 1}(\ell-D \ell)}\left(V_{S, n, 1}-\rho_{n, 1} I_{S, n, 1}\right)\right\} \\
& +\left(\alpha-\alpha^{2}\right) \\
& \times\left\{\frac{1}{2} e^{-\gamma_{n, 2}(\ell-D \ell)}\left(V_{S, n, 2}+\rho_{n, 2} I_{S, n, 2}\right)\right. \\
& \left.\quad+\frac{1}{2} e^{\gamma_{n, 2}(\ell-D \ell)}\left(V_{S, n, 2}-\rho_{n, 2} I_{S, n, 2}\right)\right\}
\end{aligned}
$$

where $\alpha=\exp (j 2 \pi / 3)$. Combining (16) and (4), one can obtain the following equation:

$$
\left|V_{d f, n}\right|=\left(\frac{4}{n \pi}\right) V_{a} .
$$

Taking the third, fifth, seventh, and ninth harmonic phasors into account, (17) determines four linear equations in one unknown and can be written in matrix form shown in (18)

$$
\begin{aligned}
& {\left[\begin{array}{llll}
\left|V_{d f, 3}\right| & \left|V_{d f, 5}\right| & \left|V_{d f, 7}\right| & \left|V_{d f, 9}\right|
\end{array}\right]^{t}} \\
& \quad=\left[\begin{array}{llll}
\left(\frac{4}{3 \pi}\right) & \left(\frac{4}{5 \pi}\right) & \left(\frac{4}{7 \pi}\right) & \left(\frac{4}{9 \pi}\right)
\end{array}\right]^{t} V_{a}+[\hat{\varepsilon}] .
\end{aligned}
$$

The unknown $V_{a}$ can also be estimated by means of the LES method. Furthermore, it is worth being mentioned that (18) is equivalent to the equation for arcing fault discrimination given in [8]. It provides the useful idea for the derivation of (18). 


\section{F. Extended DFT Algorithm}

In order to eliminate the error caused by exponentially decaying dc offset on fundamental and harmonic phasor computations, an extended DFT (EDFT) algorithm is presented in this subsection.

Consider a waveform $x(t)$ containing fundamental component, harmonics, and exponentially decaying dc offset as the following:

$$
x(t)=\sum_{n=0}^{(N / 2)-1} A_{n} \cos \left(2 n \pi f t+\theta_{n}\right)+B e^{-\alpha t}
$$

where

$n \quad$ harmonic order;

$A_{n} \quad$ amplitude of the $n$th harmonic;

$\theta_{n} \quad$ phase angle of the $n$th harmonic;

$f \quad$ fundamental frequency;

$B e^{-\alpha t}$ exponentially decaying dc offset.

Suppose that $x(t)$ is sampled with a sampling rate $(f * N)$ to produce the sample set $x(k)$ as following:

$$
x(k)=\sum_{n=0}^{(N / 2)-1} A_{n} \cos \left(\frac{2 n \pi k}{N+\theta_{n}}\right)+B e^{-\alpha(k / f N)}
$$

where $k=0.1 .2 .3 \ldots$

Taking DFT to $x(k)$ in the $r$ th moving window, one can obtain

$$
\begin{aligned}
Y_{r, n} & =\frac{2}{N} \sum_{k=0}^{N-1} x(k+r) e^{-j(2 \pi k / N) n} \\
& =A_{r, n}+B_{r, n}
\end{aligned}
$$

where $n=1,2,3 \ldots$ are harmonic order, and

$$
\begin{aligned}
A_{r, n} & =\frac{2}{N} \sum_{k=0}^{N-1} \sum_{n=0}^{(N / 2)-1} A_{n} \cos \left(\frac{2 n \pi(k+r)}{N+\theta_{n}}\right) e^{-j(2 \pi k / N) n} \\
& =A_{n} e^{j \theta_{n}} e^{j(2 \pi / N) n r} \\
B_{r, n} & =\frac{2}{N} \sum_{k=0}^{N-1} B e^{-\alpha((k+r) / f N)} e^{-j(2 \pi k / N) n} \\
& =\frac{2}{N} B e^{-\alpha(r / f N)} \sum_{k=0}^{N-1} e^{-\alpha(k / f N)} e^{-j(2 \pi k / N) n}
\end{aligned}
$$

In (21)-(23), $A_{r, n}$ is the correct phasor of the $n$th harmonic and $B_{r, n}$ is the error caused by exponentially decaying dc offset on the phasor computation of the $n$th harmonic. Conventional DFT incurs error on the fundamental and harmonic phasor computations when exponentially decaying dc offset appears. Taking observation on (21)-(23), one can find the following relationships:

$$
\begin{aligned}
& A_{r+1, n}=a_{n} A_{r, n} \\
& B_{r+1, n}=d B_{r, n}
\end{aligned}
$$

where

$$
a_{n}=e^{j(2 \pi / N) n} ; \quad d=e^{-\alpha(1 / f N)}
$$

and

$$
\begin{aligned}
& Y_{r+1, n}=a_{n} A_{r, n}+d B_{r, n} \\
& Y_{r+2, n}=a_{n}^{2} A_{r, n}+d^{2} B_{r, n} .
\end{aligned}
$$

It is notable that the $d$ in (25) is independent of variable $n$. So $d$ is a constant for every harmonic. Taking some algebraic manipulations in (21), (26), and (27) on the fundamental frequency component, one can obtain

$$
d=\frac{\left(a_{1} Y_{r+1,1}-Y_{r+2,1}\right)}{\left(a_{1} Y_{r, 1}-Y_{r+1,1}\right)}
$$

According to (26) and (28), one can obtain correct phasors of fundamental component and harmonics in the $r$ th moving window as the following:

$$
A_{r, n}=\frac{\left(d Y_{r, n}-Y_{r+1, n}\right)}{\left(d-a_{n}\right)} .
$$

\section{CONCLUSION}

In part I of this two-paper set, we present the theory and algorithms for the proposed technique. The proposed fault detection/location technique by processing the synchronized fundamental voltage and current phasors will be utilized to both arcing and permanent faults, and the proposed arcing fault discrimination technique by processing the synchronized harmonic voltage and current phasors is to avoid reclosing on a permanent fault. In this paper, an extended DFT algorithm is also presented to eliminate the error caused by exponentially decaying dc offset on the computations of fundamental and harmonic phasors. In this study, a great deal of computer simulations by Matab ${ }^{\circledR} /$ power System Blockset simulator has been done for performance evaluation on the proposed technique. These simulation results and a test case using real-life measured data will be presented in the companion paper.

\section{REFERENCES}

[1] P. M. Anderson, Power System Protection. New York: McGraw-Hill, 1999.

[2] A. G. Phadke and J. S. Thorp, Computer Relaying for Power System. New York: Wiley, 1988.

[3] A. T. Johns and S. K. Salma, Digital Protection for Power System. Stevenage, U.K.: Peregrinus, 1995.

[4] T. Takagi, T. Jamakoshi, M. Yamaura, R. Kondow, and T. Matshusima, "Development of a new type fault locator using one-terminal voltage and current data," IEEE Trans. Power App. Syst., vol. PAS-101, pp. 2892-2898, Aug. 1982.

[5] D. J. Lawrence, L. Z. Cabeza, and L. T. Hochbert, "Development of an advanced transmission line fault location system, part II. Algorithm development and simulation," IEEE Trans. Power Delivery, vol. 7, pp. 1972-1981, Oct. 1992.

[6] M. Akke and J. S. Thorp, "Some improvements in the three-phase differential equation algorithm for fast transmission line protection," IEEE Trans. Power Delivery, vol. 13, pp. 66-72, Jan. 1998.

[7] M. B. Djuric, Z. M. Radojevic, and V. V. Terzija, "Time domain solution of fault distance estimation and arcing faults detection on overhead lines," IEEE Trans. Power Delivery, vol. 14, pp. 60-67, Jan. 1999.

[8] M. B. Djuric and V. V. Terzija, "A new approach to the arcing faults detection for fast autoreclosure in transmission systems," IEEE Trans. Power Delivery, vol. 10, pp. 1793-1798, Oct. 1995.

[9] Y. Ge, F. Sui, and Y. Xiao, "Prediction methods for preventing singlephase reclosing on permanent fault," IEEE Trans. Power Delivery, vol. 4, pp. 114-121, Jan. 1989. 
[10] R. K. Aggarawl, A. T. Johns, Y. H. Song, R. W. Dunn, and D. S. Fitton, "Neutral-network based adaptive single-pole autoreclosure technique for EHV transmission systems," Proc. Inst. Elect. Eng., Gen. Transm. Dist., vol. 141, no. 2, pp. 155-160, Mar. 1994.

[11] J.-A. Jiang, J.-Z. Yang, Y.-H. Lin, C.-W. Liu, and J.-C. Ma, “An adaptive PMU based fault detection/ location technique for transmission lines: Part I: Theory and algorithms," IEEE Trans. Power Delivery, vol. 15, pp. 486-493, Apr. 2000.

[12] J.-A. Jiang, Y.-H. Lin, J.-Z. Yang, T.-M. Too, and C.-W. Liu, “An adaptive PMU based fault detection/ location technique for transmission lines: Part II: PMU implementation and performance evaluations," IEEE Trans. Power Delivery, vol. 15, pp. 1136-1146, Oct. 2000.

[13] A. T. Johns, R. K. Aggarawl, and Y. H. Song, "Improved techniques for modeling fault arcs on faulted EHV transmission system," Proc. Inst. Elect. Eng., Gen. Transm. Dist., vol. 141, no. 2, pp. 148-154, Mar. 1994.

[14] M. Kizilcay and K. H. Koch, "Numerical fault arc simulation based on power arc test," Eur. Trans. Elect. Power Eng., vol. 4, no. 3, pp. 177-185, May/June 1994

[15] Y. Goda, M. Iwata, K. Ikeda, and S. I. Tanaka, "Arc voltage characteristics of high current fault arcs in long gaps," IEEE Trans. Power Delivery, vol. 15, pp. 791-795, Apr. 2000.

[16] H. W. Dommel, EMTP Theory Book. Vancouver, BC, Canada: Microtran Power System Analysis Corp., 1992, pp. 4-50-4-56.

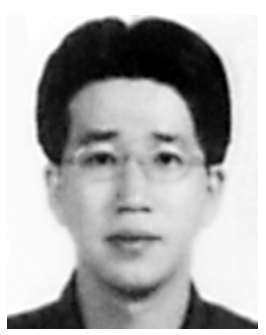

Ying-Hong Lin (S'01-M'03) was born in Taipei, Taiwan, R.O.C., in 1970. He received the B.S. degree in electrical engineering from Taiwan University of Technology and Science, Taipei, in 1995 and the M.S. and Ph.D. degrees in the electrical engineering from National Taiwan University, Taipei, in 1999 and 2002, respectievly.

Currently, he is an Assistant Professor in the Department of Engineering and Management of Advanced Technology, Chang Jung Christian University, Tainan, Taiwan. His research interests are the application of global positioning systems (GPS) and phasor-measurement units (PMUs) in power system protection and very-large scale integrated (VLSI) design.

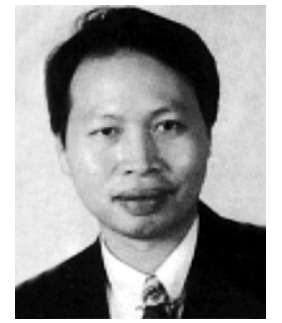

Chih-Wen Liu (S'93-M'96-SM'03) was born in Taiwan, R.O.C., in 1964. He received the B.S. degree in electrical engineering from National Taiwan University, Taipei, in 1987 and the M.S. and $\mathrm{Ph} . \mathrm{D}$. degrees in electrical engineering from Cornell University, Ithaca, NY, in 1992 and 1994 respectively.

Currently, he is Professor of electrical engineering with National Taiwan University, where he has been since 1994. His research interests include application of computer technology to power system monitoring, operation, protection, and control. Other research interests include motor control and power electronics.

Dr. Liu is a Reviewer for the IEEE TRANSACTIONS ON POWER SYSTEMS and the IEEE TRANSACTIONS ON POWER DELIVERY.

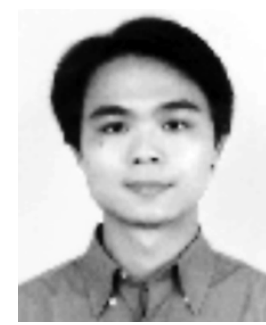

Ching-Shan Chen (S'01) was born in Taichung, Taiwan, R.O.C., in 1976. He received the B.S. degree in electrical engineering from National Taiwan University of Technology and Science, Taipei, in 1998 and the M.S. and Ph.D. degrees in electrical engineering from National Taiwan University, Taipei, in 2000 and 2003, respectively.

His research interests include computer relaying and the application of phasor-measurement units (PMUs) to power system protection. 